\author{
Bryce Morsky* and Erol Akçay ${ }^{\dagger}$ \\ University of Pennsylvania
}

\title{
False beliefs can bootstrap cooperative communities through social norms
}

October 2, 2020

\begin{abstract}
Building cooperative communities has always been a crucial problem for human societies. Much research suggests that cooperation is facilitated by knowing who exactly is a cooperator and defector, and being able to respond accordingly. As such, anonymous games is thought to hinder cooperation. Here, we show that this conclusion is altered dramatically in the presence of conditional cooperation norms and heterogeneous beliefs about others' behaviours. Specifically, we show that inaccurate beliefs about other players' behaviours can in fact help foster and stabilize cooperation via social norms. To show this, we combine the population dynamics of a social community with the game theory of interactions within the community. In our model, individuals can join a community based on beliefs generated by public signals regarding the level of cooperation within, and decide to cooperate or not depending on these beliefs. These signals may overstate how much cooperation there really is. We show that even if individuals eventually learn the true level of cooperation, the initially false beliefs can trigger a dynamic that sustains high level of cooperation within the community. We also characterize how the rates of joining, leaving, and learning in the community affect the cooperation level and community size simultaneously. Our results illustrate how false beliefs in the presence of conditional cooperation norms can help build up cooperative communities.
\end{abstract}

Keywords: community emergence, conditional cooperation, expectations, social norms

*morsky@sas.upenn.edu

†eakcay@sas.upenn.edu 


\section{Introduction}

The ecological and evolutionary success of our species is in large part dependent on our ability to start and grow cooperative communities where individuals work together for common aims beyond their immediate kin. Humans are exceptionally good at cooperating with one another, and understanding the underpinnings of such cooperation has been a primary goal of evolutionary human sciences for decades. Extensive experimental evidence shows that human cooperation (and any other social behavior) is contingent and is affected by others' behaviors (Fischbacher et al., 2001; Frey and Meier, 2004; Chaudhuri, 2011), and social norms about own and others' behaviors (Bicchieri and Chavez, 2010; Kimbrough and Vostroknutov, 2016; Bicchieri et al., 2020a).

Social norms can be defined as the rules of the game in social interactions (Bicchieri. 2006). Many norms prescribe what norms holders should do given what others do or what situations individuals encounter. The content of these norms evolve culturally (Gelfand and Jackson, 2016), yet humans have innate predisposition for internalizing culturally acquired norms (Gintis, 2003). Such social norms can instigate group-beneficial behaviors (Gintis, 2003; Gavrilets and Richerson, 2017; Akçay and Van Cleve, 2019) or coordinate social behaviors for mutual gain (Gintis, 2010; Morsky and Akçay, 2019). Importantly, adopting a social norm does not mean that individuals blindly follow them: rather, social norms frequently act to shape preferences or default behaviors of individuals, who still make decisions that are responsive to their payoffs or other signals and beliefs. For example, an important social norm is conditional cooperation (Bicchieri, 2010; Traxler and Spichtig, 2011; Bicchieri et al., 2020b), wherein norm holders will cooperate if they believe that others are cooperating. This kind of conditional behavior can be generated by individuals having "relational utilities" from cooperating that make cooperation more rewarding (or non-cooperation more aversive) if they know or expect others to cooperate. If the proportion of such norm holders is high, then this reciprocity norm can sustain cooperation.

Building a cooperative community by relying on reciprocity norms thus faces a catch22: people will cooperate only if they believe others will, too. Getting such communities off the ground is a problem that human societies had to solve over and over again. This problem is very salient in contemporary life in online communities, for example, filesharing software that rely on users' willingness to provide uploads of files for others. A solution to this problem that arose in this context is termed charismatic code (Strahilevitz, 2003). Charismatic code is technology that exploits social mechanisms to induce cooperation amongst its users. It can distort viewers' normative expectations by masking cheating, exaggerating levels of cooperation, and promoting collective identity and prosocial norms. 
For example, the file-sharing software Morpheus would depict the total number of users and files being shared, which suggested a very high share rate per person (Strahilevitz. 2003). However, this hid the underlying heterogeneity in sharing where the majority of users shared relatively little. This omitted truth portrayed a cooperative community in which everyone gave back what they took, which in turn fuelled internal norms of reciprocity and conditional cooperation thereby stimulating more cooperation than there otherwise would be. Charismatic code therefore provides an explanation for why there can be substantial trust and cooperation in anonymous groups of file-sharers.

This phenomenon can be seen in other contexts as well (Strahilevitz, 2003). An example is binge drinking norms (Perkins, 1997; Lewis and Neighbors, 2006), which can be fostered by conspicuous signalling and conditional following of the norm (here the norm is drinking). Students often overestimate the level of binge drinking and thus drink more than they otherwise would. Those who are heavily intoxicated are likely to be more visible than those not, which contributes to this. Thus, these beliefs can become self fulfilling prophecies. Understanding this, campus programs that publicize the lower than expected levels of binge drinking have lowered the prevalence of it (Haines, 1996). In a similar way, publicizing compliance can increase compliance via conditional cooperation with respect to tax compliance (Coleman, 1996). The interplay between expectations and norms also has implications for vaccination decision making (Xia and Liu, 2014).

Our goal in this manuscript is to understand how distortions in the signals individuals are getting about others' behaviors can affect the dynamics of conditional cooperation norms. Specifically, we are interested in when such distortions can get cooperative communities started and maintain them. To explore these questions, we build a mathematical model of the growth and decline of a community as individuals join and leave it, as well as the joint dynamics of individual behavior and beliefs as a consequence of (potentially distorted) signals and individual learning.

We model agents within the community that earn material utility from a public goods game, as well as experiencing "relational utility" which represents internalized conditional cooperation norms (Traxler and Spichtig, 2011; Gavrilets and Richerson, 2017; Akçay and Van Cleve, 2019). Specifically, we assume agents experience utility ("warm glow") or disutility ("guilt") based on their own cooperation level relative to their perception of the mean cooperation level in the community. Depending on the strength of this relational utility, each individual has a threshold perceived level of cooperation at which they too cooperate. Players inside the community can further learn - through their own experience and interacting with other players - the true level of cooperation within the community and base their behaviour on this. Those outside of the community also have a perception 
of the level of cooperation within the community, though this is only through signalling from those within the community. These outsiders may then enter the community with that belief determining the level of cooperation they will engage in, while insiders who have learned the true level of cooperation can become discouraged and leave the community. We show that the relative rates of entering communities, learning the true level of cooperation, and leaving determine whether a cooperative community can exist and how much cooperation can be sustained.

\section{Model}

\subsection{Basic conditional cooperation model}

We begin with a model of a public goods game with a norm of conditional cooperation from (Traxler and Spichtig, 2011), in which individuals cooperate (donate to a public good) if a sufficient proportion of the community cooperates (see Appendix SI-1 for details). Though we will frame our discussion in terms of cooperation, our model applies more generally to conditional adherence to a behaviour. We assume that the sensitivity to the norm varies within the population. Let $F(p)$ be the cumulative density function of this norm sensitivity, i.e. $F(p)$ is the proportion of the population that will cooperate given that proportion $p$ of the community is cooperating. We first assume here that the real and perceived levels of cooperation are the same. Later, we will consider the case where they are not by adding belief dynamics.

We consider an evolutionary model wherein individuals will change their behaviour relative to the difference between their normative value and the level of cooperation. Thus, the dynamics are determined by the equation $\dot{p}=F(p)-p$. The equilibria are $p^{*}=F\left(p^{*}\right)$, and stability is found for $F^{\prime}\left(p^{*}\right)=f\left(p^{*}\right)<1$, and instability for $f\left(p^{*}\right)>1$. There is at least one stable equilibrium (which for parameters we are concerned with will be for low cooperation). In addition to this equilibrium, there may be an unstable one, which is repelling for $p<p^{*}$ and attracting for $p>p^{*}$. Finally, there may also be three equilibria: stable low cooperation, unstable intermediate cooperation, and stable high cooperation, relatively speaking. Figure 1 d depicts both the three and one equilibria cases, which we will denote the coordination dilemma (blue curve) and the cooperation dilemma (red curve), respectively. The type of dilemma switches between these depending on the parameters of $F(p)$ curve. This model is our base setting. Below, we show that distorting the signals about the behavior within the community can alter these equilibria to maintain a stable community with high levels of cooperation. 
(a)

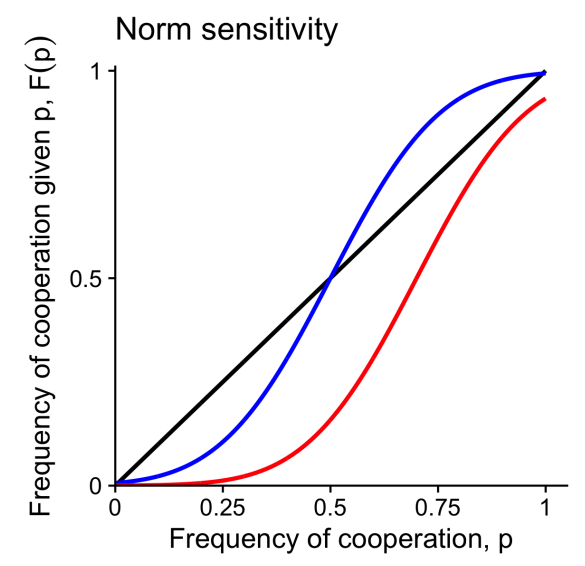

(b)

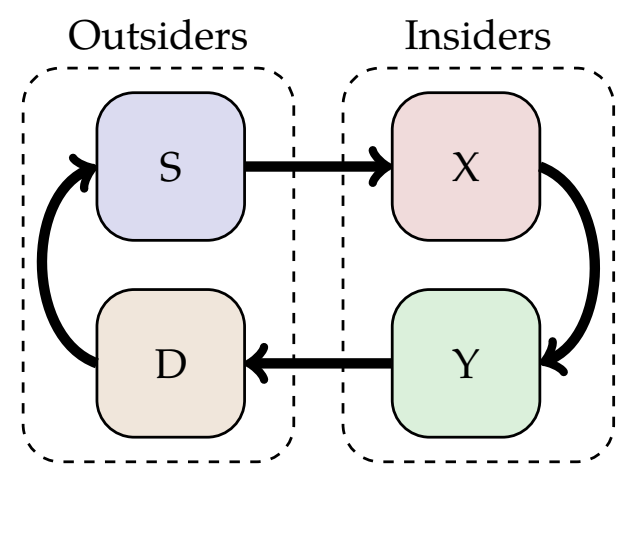

Figure 1: (a) The frequency of cooperation given that a proportion $p$ are cooperating for two different distributions of norm sensitivity. Here, $F(p)$ is the cumulative distribution function of a normal distribution with means $\mu=0.5$ and 0.7 for the coordination (blue curve) and cooperation (red curve) dilemmas, respectively; the variance is $\sigma^{2}=0.04$ for both. For the coordination dilemma, we have a bistable system with stable states of high cooperation and low cooperation. For the cooperation dilemma, we have a sole stable fixed point with low cooperation. Note here that there is no deception or misinformation; players know the true level of cooperation. (b) Diagram of the four compartment model. Susceptible players, $S$, enter the community as new naive players, $X$. Through community interactions, they may become savvy players, $Y$, who know the true level of cooperation. If there is a discrepancy between the true level of cooperation and the belief $\hat{q}, Y$ players may become discouraged, $D$. Discouraged players then become susceptible (again).

\subsection{Model of community and belief dynamics}

We consider a compartmental model (as depicted in Figure $1 \mathrm{~b}$ ) with four classes of individuals: two currently belonging to the community in which the public goods game is played (insiders), and two that do not belong to it (outsiders). Individuals can transition between these classes as depicted in Figure $1 \mathrm{~b}$.

Specifically, insiders belong to two classes: "naive" and "savvy" with $X$ and $Y$ denoting the numbers of these insiders, respectively, and $I=X+Y$ denoting the total number of insiders. Naive insiders have a belief $\hat{q}$ about the level of cooperation in the community, and thus a group of them will cooperate at level $q=F(\hat{q})$. We assume that naive insiders eventually learn the true level of cooperation in the population and therefore become savvy by interacting with other community members and observing their behaviours. The rate at which naive insiders become savvy is determined by both a learning rate, $\ell>0$, and the difference between the beliefs of the naive individuals and the behaviour of those they interact with. Therefore, we consider a flow $\ell((\hat{q}-q) X+(\hat{q}-p) Y) / I=\ell(\hat{q}-\bar{p}) X$ 
where $\bar{p}=y p+(1-y) q$ is the mean (actual) level of cooperation, $y=Y / I$ is the frequency of savvy insiders, and $p$ is the mean cooperation level of savvy insiders. The dynamics of $p$ are then determined by

$$
\dot{p}=F(\bar{p})-p .
$$

Next, we consider a group of outsiders that are not a part of the community, and thus do not play its public goods game and are ignorant of $\bar{p}$. If the total population of insiders and outsiders is $K$, then the number of outsiders is $K-I$. Among the outsiders, there is a group, $S$, who are susceptible to enter the community when interacting with community members. These outsiders enter the community as new naive members at a rate proportional to their contact rate with community members: $\iota S I / K$ for inflow rate $\iota>0$. Conversely, we assume that savvy insiders may become discouraged with the community and may leave at a rate proportional to the difference $\hat{q}-\bar{p}$, i.e. $Y$ flows out of the community at rate $\omega(\hat{q}-\bar{p}) Y$ for outflow rate $\omega>0$. Note that we will assume that $p$ is bounded above by $\hat{q}$.

Savvy players may leave the community discouraged that the level of cooperation does not meet the naive expectation, $\hat{q}$. These discouraged outsiders, $D$, are not directly susceptible to reentry. However, they can become susceptible by loss of their discouragement, their confidence in $\bar{p}$, or their memory of $\bar{p}$. The parameter $\varphi$, the rate at which $D$ flows to $S$, encapsulates all of these possible mechanisms. Considering the model in terms of $S, I$, $y$, and $p$, the dynamics of the system are described by the set of equations:

$$
\left\{\begin{array}{l}
\dot{S}=\varphi(K-S-I)-\iota I \frac{S}{K}, \\
\dot{I}=\iota I \frac{S}{K}-\omega(\hat{q}-\bar{p}) y I, \\
\dot{y}=(\hat{q}-\bar{p})(1-y)(\ell-\omega y)-\iota y \frac{S}{K}, \\
\dot{p}=F(\bar{p})-p .
\end{array}\right.
$$

See Appendix SI-2.1 for the full derivation.

Throughout, we consider two case: the coordination and cooperation dilemmas where $q=F(\hat{q})=\hat{q}$ and $q=F(\hat{q})<\hat{q}$, respectively. We determine the joint equilibria for insider and outsider populations, their compositions, and the cooperation level amongst the insiders. In doing so we explore the parameters, as summarized in Table 1, to see how they impact the qualitative behaviour of the model. 


\begin{tabular}{cl}
\hline Parameter & Description \\
\hline$\mu$ & Mean norm sensitivity \\
$\sigma^{2}$ & Variance in norm sensitivity \\
$\iota$ & Inflow rate \\
$\ell$ & Learning rate \\
$\omega$ & Outflow rate \\
$\varphi$ & Resusceptiblity rate \\
\hline
\end{tabular}

Table 1: Summary definitions of parameters.

\section{Results}

\subsection{Coordination dilemma}

We first consider a coordination dilemma, which is characterized by three equilibria in the basic model without community dynamics. In the community dynamics model, the high cooperation equilibrium is always a stable equilibrium set where all players are in the community and are cooperating at the same high level, i.e. $S^{*}=0, I^{*}=K$, and $p^{*}=q=\hat{q}$. As naive and savvy players are cooperating at the same rates, there is no difference between them and all values of $y^{*}$ can be an equilibrium (see Appendix SI-2.2 Theorem 11).

Whether or not we have more equilibria depends on two ratios: the ratio of leaving to learning rates, $\omega / \ell$, and the ratio of inflow to outflow,

$$
\mathcal{R}_{0}(y, p)=\frac{\iota}{\omega\left(\hat{q}-\bar{p}^{*}\right) y^{*}},
$$

evaluated at equilibrium $y^{*}$ and $\bar{p}^{*}$. $\mathcal{R}_{0}$ effectively measures whether the community will grow from zero, analogous to the concept of the basic reproduction number in epidemiology.

At a low ratio of leaving to learning rates, $\omega / \ell$, there may be two more equilibria: one stable with low cooperation, and another unstable with moderate cooperation (see below for more on stability of these equilibria). These equilibria come from the number of solutions to the dynamic of savvy players' behaviours, $\dot{p}$, i.e. solutions to $p=F(\bar{p})$. Further, it is necessary that $y^{*} f\left(\bar{p}^{*}\right)<1$ for them to be stable (see Appendix SI-2.2. As such, the results we describe here are necessary conditions for an equilibrium to exist and be stable, but not sufficient. As $\omega / \ell$ increases, these two solutions converge until they annihilate each other, leaving only the high-cooperation equilibrium described before. Thus, by decreasing the rate of learning by naive individuals or increasing the rate at which savvy 
(a)

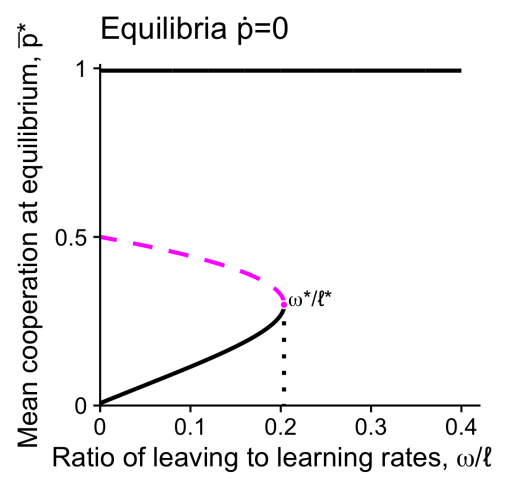

(b)

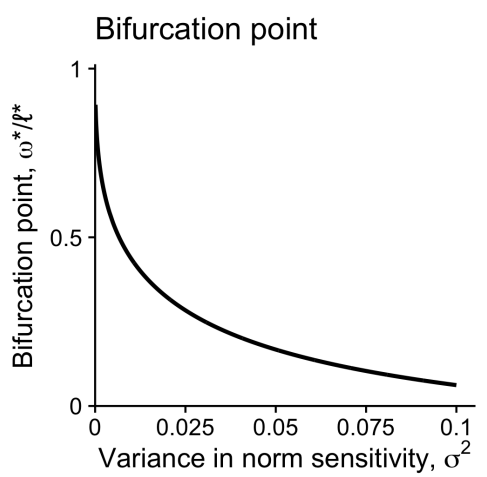

(c)

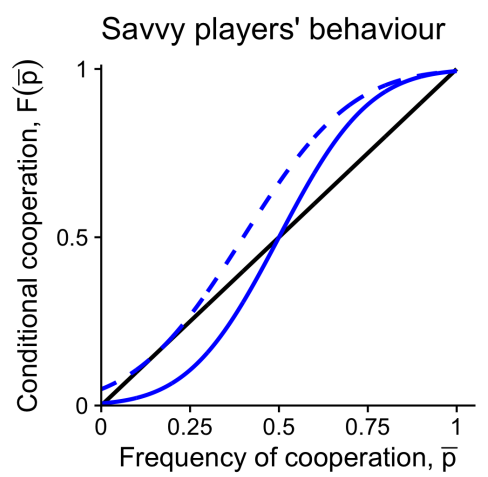

Figure 2: (a) Increasing $\omega$ relative to $\ell$ annihilates the lower and medium equilibria of $p=F(\bar{p})$ leaving only the high equilibrium $p^{*}=q$. The solid and magenta dashed curves represent the stable and unstable equilibria, respectively. (b) The bifurcation point is decreasing for increasing variance in the norm sensitivity. (c) The presence of naive individuals shifts the solid blue curve up to the dashed blue curve. At this bifurcation point, the low and middle $p^{*}$ equilibria are destroyed. $F$ is the $\mathrm{CDF}$ of $\mathcal{N}(0.5,0.04)$.

individuals leave, a low cooperation community can suddenly become a high cooperation community (Figure 2).

How this transition happens can be seen by first considering the proportion of savvy insiders at equilibrium. Provided that both the insider and outsider communities are nonzero at equilibrium, the equilibrium proportion of savvy insiders is $y^{*}=\ell /(\ell+\omega) \cdot y^{*}$ will decrease as $\omega / \ell$ increases as savvy individuals leave faster than naive individuals become savvy. Because naive individuals cooperate at higher levels than savvy individuals, this increases the average level of cooperation in the community, in turn inducing more savvy individuals to cooperate as well. This means that as $\omega / \ell$ increases, the blue curve $F(\bar{p})$ from Figure $1 \mathrm{a}$ is shifted up, as depicted in Figure $2 \mathrm{c}$. The higher $\omega / \ell$, the lower the fraction of savvy individuals at equilibrium, and the more the curve will be shifted upwards. For a sufficiently large shift upwards, all but the high equilibrium can be annihilated. Figure $2 \mathrm{a}$ shows that as the ratio of the leaving to learning rate increases, the intermediate equilibrium of $\dot{p}$ decreases while the low-cooperation equilibrium increases. These two equilibria meet and annihilate one another at a saddle-node bifurcation where $p^{*}=F\left(\bar{p}^{*}\right)$ is tangent to the diagonal.

Interestingly, the bifurcation point decreases as the variance in norm sensitivity increases, as Figure $2 b$ shows. As the variance increases, the lowest equilibrium $p^{*}$ increases towards the middle equilibrium. As such, the curve needs to be shifted up less to reach the bifurcation. In other words, highly cooperative communities can be globally stable at higher learning (or lower leaving) rates. 
Note that the stability of these equilibria and whether or not the equilibria for the other compartments exist will depend on $\mathcal{R}_{0}$ and $f(\bar{p})$. In particular, we can write the mixed equilibrium of insiders and outsiders as functions of $\mathcal{R}_{0}$ :

$$
S^{*}=\frac{K}{\mathcal{R}_{0}}, \quad I^{*}=K \frac{\mathcal{R}_{0}-1}{\mathcal{R}_{0}+\iota / \varphi},
$$

which implies that a polymorphic equilibrium exists only if $\mathcal{R}_{0}>1$. A further necessary condition for stability is that $y^{*} f\left(\bar{p}^{*}\right)<1$ (see Appendix SI-2.2).

(a)

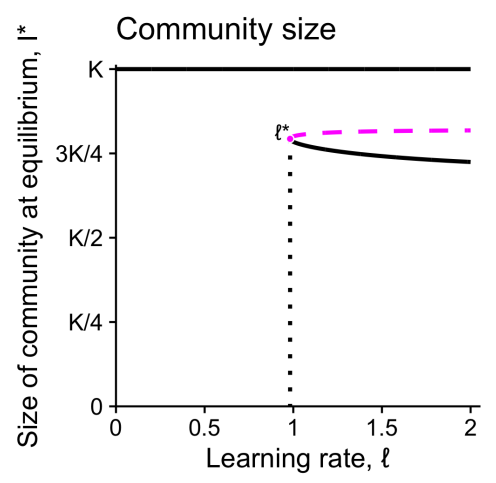

(c)

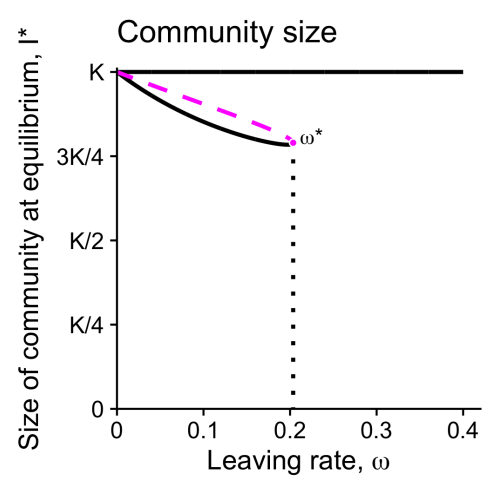

(b)

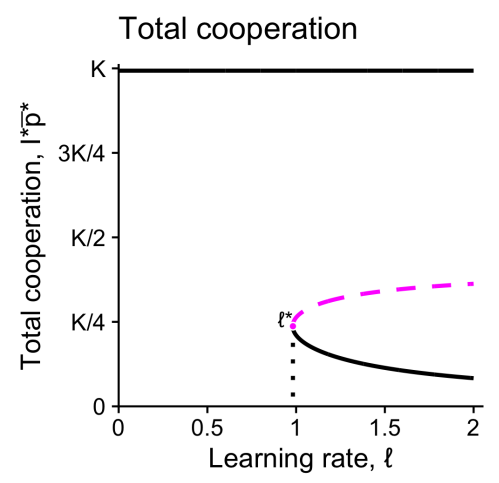

(d)

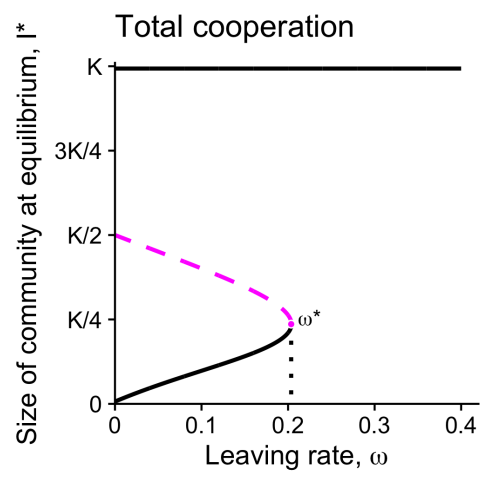

Figure 3: $(a, b)$ Increasing $\ell$ causes a blue sky bifurcation in both the community size and total cooperation. Here the parameters are $\iota=\varphi=1$ and $\omega=0.2$. (c, d) Increasing $\omega$ will reduce the community size yet increase the total amount of cooperation. At a bifurcation point, the low and middle equilibria are destroyed. Here, $\iota=\omega=\varphi=1$. The solid and magenta dashed curves represent the stable and unstable equilibria, respectively. $F$ is the CDF of $\mathcal{N}(0.5,0.04)$. the

Next, we consider the impact of the parameters on the community size, $I^{*}$, and total amount of cooperation across all individuals. Since $\partial I^{*} / \partial \iota>0$ and $\partial I^{*} / \partial \varphi>0, I^{*}$ increases with respect to increasing $\iota$ and $\varphi$. Further, since $y^{*}$ and $p^{*}$ are not affected by these parameters, increasing $I^{*}$ will increase the total amount of cooperation in the popu- 
lation. However, the impact of $\ell$ and $\omega$ are more complicated. Figures $3 \mathrm{a}$ and $3 \mathrm{~b}$ show that for low $\ell$, there is no polymorphic equilibrium of insiders and outsiders; the entire population will be in the community. Increasing $\ell$ produces a blue sky bifurcation in which there are two polymorphic equilibria: one unstable, and one stable with a relatively lower community size and total level of cooperation. Figures $3 \mathrm{c}$ and $3 \mathrm{~d}$ depict the results for varying $\omega$. For low and high $\omega$, we observe three equilibria and one equilibria, respectively. Up to the bifurcation point, increasing $\omega$ reduces the community size of both polymorphic equilibria. However, it increases the total level of cooperation for the stable equilibrium and decreases it for the unstable one.

\subsection{Cooperation dilemma}

Now let us consider the cooperation dilemma where $q=F(\hat{q})<\hat{q}$. Here again, the ratio of the leaving to learning rates, $\omega / \ell$, determines how the equilibria will behave (Figures 4 a 4c), but this behavior is modulated qualitatively by the variance in norm sensitivity. For low variance (Figure 4a), we actually observe a coordination dilemma, with the same patterns as above. However, for higher variance (Figure 4b), we initially have a single equilibrium with low cooperation. The cooperation level $\bar{p}^{*}$ increases with $\omega / \ell$, until a pitchfork bifurcation at which two more equilibria are created. One of these is a high cooperation equilibrium and stable, and the other intermediate cooperation and unstable. Further increasing $\omega / \ell$ causes the latter equilibrium collide with and annihilate the low cooperation equilibrium, leaving us with only one stable, high cooperation equilibrium, although this one still falls short of the aspirational cooperation rate $\hat{q}$. For yet higher variance, $\bar{p}^{*}$ increases smoothly with $\omega / \ell$ (Figure $4 c$ ). In this scenario, the aspirational cooperation rate $\hat{q}$ is not sustainable nor is there a discrete jump in cooperation. Yet we can increase average cooperation at equilibrium and approach $\hat{q}$ as we increase $\omega$ relative to $\ell$, simply through increasing the proportion of naive individuals.

Figure $4 \mathrm{~d}$ summarizes the bifurcations we can observe. For low variance in norm sensitivity, we observe a coordination dilemma, and the saddle-node bifurcation point decreases as the variance increases, as in Figure $2 \mathrm{~b}$. However, at $\sigma^{2} \approx 0.025$ for these parameter values, the cooperation dilemma emerges, which features a pitchfork bifurcation. The difference between the two curves is the region of $\omega / \ell$ in which this occurs. For sufficiently large variance, the bifurcation points disappear, and the equilibrium level of cooperation is simply a monotonically increasing function of $\omega / \ell$. As in the coordination dilemma, cooperation is boosted by the presence of inaccurate beliefs about the level of cooperation shifting the norm sensitivity (reaction curve) up as depicted in Figure $4 \mathrm{e}$. 
(a)

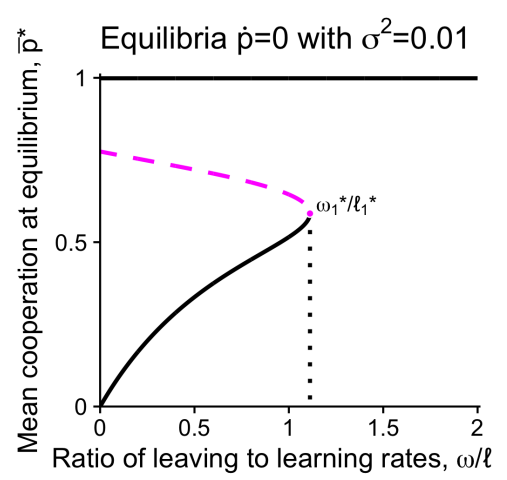

(b)

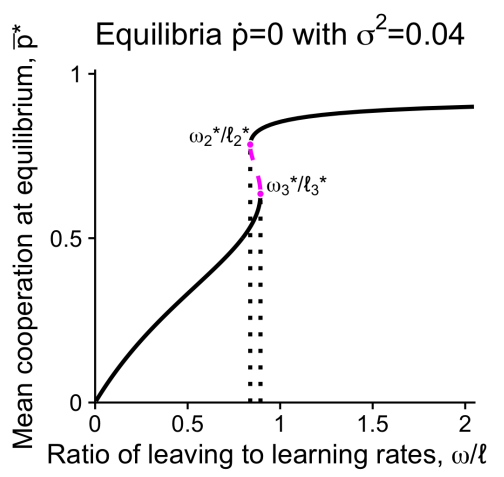

(c)

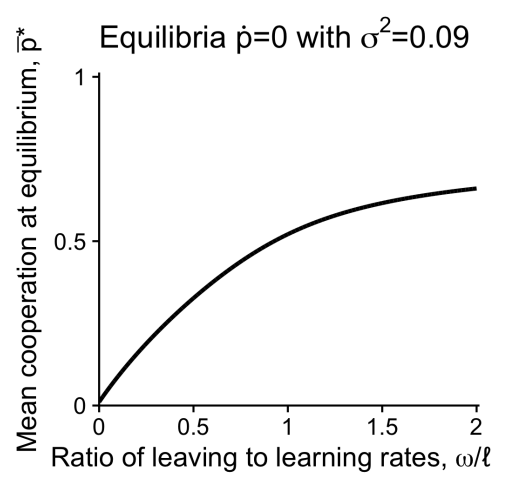

(d)

Bifurcation point

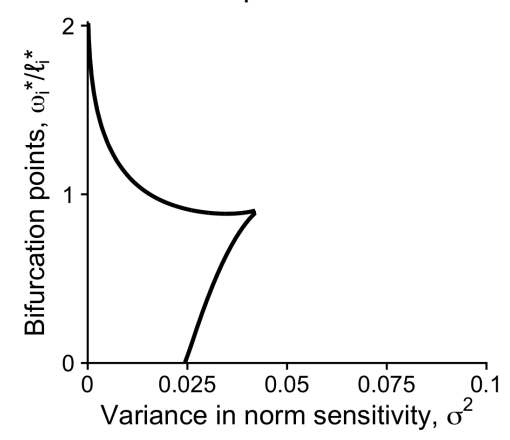

(e)

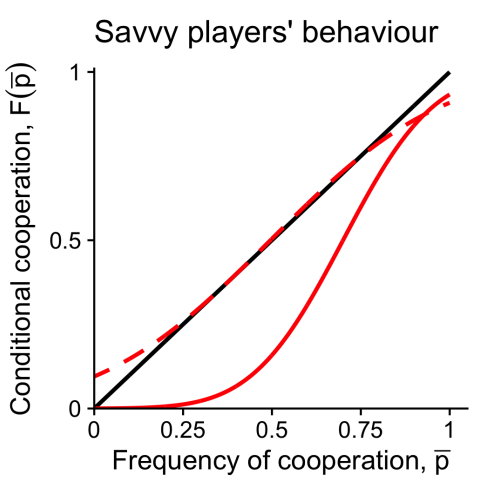

Figure 4: (a) For low variance, we observe the coordination dilemma case with saddle-node bifurcation point $\omega_{1}^{*} / \ell_{1}^{*}$. However, the qualitative behaviour of the system changes for higher variance. The solid and magenta dashed curves represent the stable and unstable equilibria, respectively. (b) Increasing $\omega$ relative to $\ell$ increases the equilibrium value of cooperation. The supercritical pitchfork bifurcation point, $\omega_{2}^{*} / \ell_{2}^{*}$, leads to three equilibria. These equilibria are destroyed at the subcritical bifurcation point $\omega_{3}^{*} / \ell_{3}^{*}$, after which the equililibrium level of cooperation continues to rise. (c) With sufficiently high variance, these bifurcations vanish, and cooperation increases as $\omega / \ell$ increases. (d) Until $\sigma^{2} \approx 0.025$, the system is a coordination dilemma as in (a), and thus the bifurcation point is decreasing for increasing variance in the norm sensitivity. As we increase $\sigma^{2}$, we have a pitchfork bifurcation with two bifurcation points as in (b) until $\sigma^{2} \approx 0.042$. For higher $\sigma^{2}$, we have no bifurcations and $\vec{p}^{*}$ is a linearly increasing function as in (c). (e) The presence of naive individuals shifts the curve up creating new equilibria (here $\left.\sigma^{2}=0.04\right)$. For all figures, $\hat{q} \approx 0.993$ and $F$ is the $\operatorname{CDF}$ of $\mathcal{N}\left(0.7, \sigma^{2}\right)$.

Figure 5 shows the impact of the learning and leaving rates on the community size and total population. Increasing $\ell$ generally reduces both the community size and total cooperation. Note that there are pitchfork bifurcations with three equilibria created and then destroyed as $\ell$ is increased (Figures $5 \mathrm{a}$ and $5 \mathrm{~b}$ ). Increasing $\omega$ from near zero will initially decrease the community size. However, the community size will increase after which a pitchfork bifurcation occurs. Further increasing $\omega$ returns the system to a single polymor- 
(a)

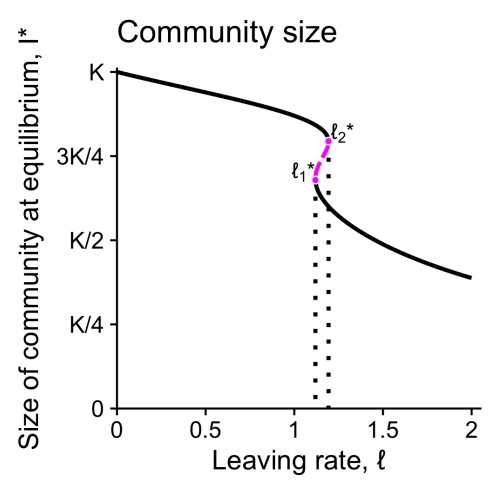

(c)

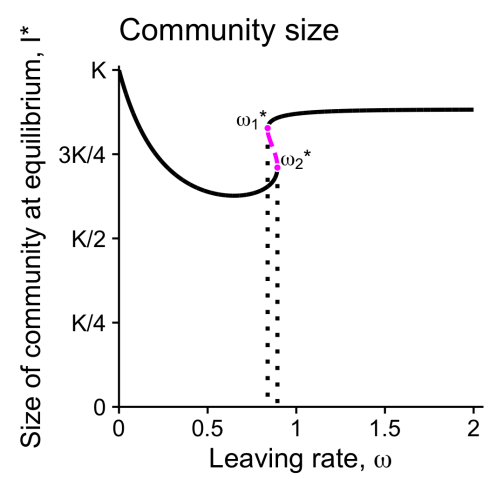

(b)

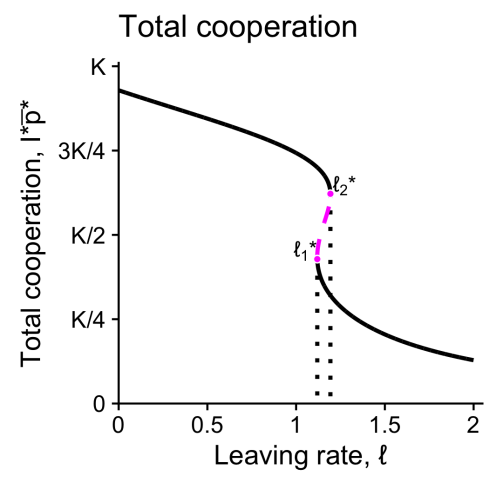

(d)

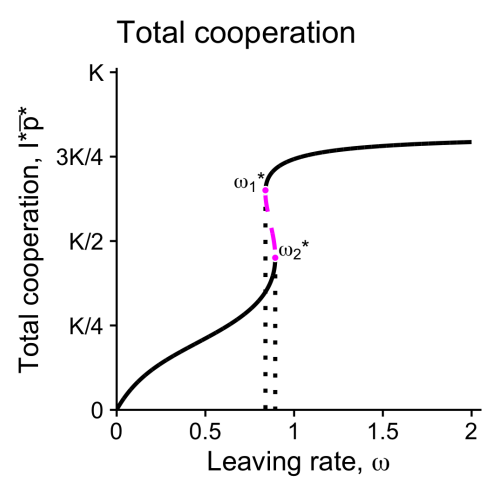

Figure 5: (a, b) Increasing $\ell$ decreases the size of the community and the total amount of cooperation. However, there is a fold bifurcation. Here the parameters are $\iota=\omega=\varphi=1$. (c) Increasing $\omega$ will initially reduce $I^{*}$ until $I^{*}$ begins to rise. A bifurcation occurs after which $I^{*}$ asymptotes. (d) Increasing $\omega$ increases the total amount of cooperation in the population. For $c$ and $\mathrm{d}, \iota=\ell=\varphi=1$. The solid and magenta dashed curves represent the stable and unstable equilibria, respectively. For all figures, $F$ is the CDF of $\mathcal{N}(0.7,0.04)$.

phic equilibrium, and the community size plateaus. The total amount of cooperation in the population, however, is increasing with $\omega$. Although, the bifurcations are also present.

\subsection{Community crash and cycling}

We next consider the conditions under which the cooperative community crashes in the cooperation dilemma. A community crash is when the number of insiders goes to zero and does not rebound. This occurs if $\mathcal{R}_{0} \leq 1$ and $f\left(\bar{p}^{*}\right)<1$ at this state (see Appendix SI-2.2. Theorem 22. Figure 6 plots the effects of different parameters on the existence of the crashing state. For low enough inflow rate $\iota$, the crashing equilibrium is stable (equivalently, $\mathcal{R}_{0}<1$; Figure 6a). However, as $\iota$ increases, a positive community size can also be stable in conjunction with the crashing equilibrium. Above a threshold $\iota^{*}$, the crashing 
(a)

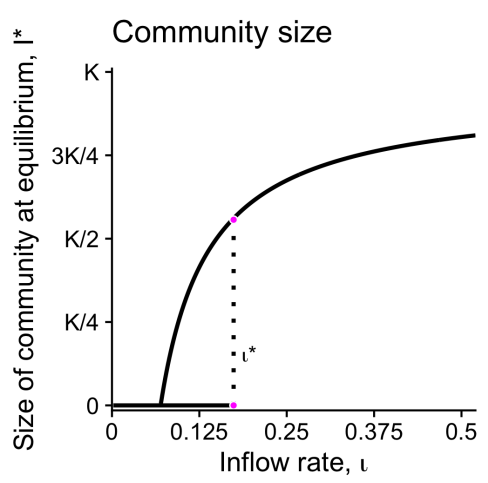

(b)

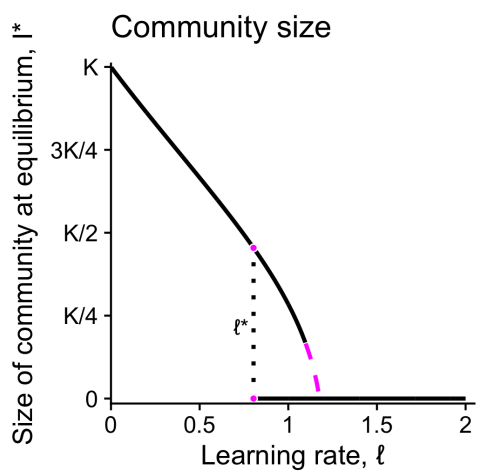

(c)

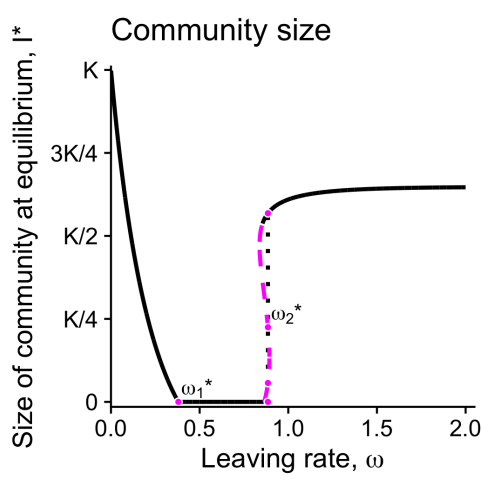

Figure 6: The parameters $\iota, \ell$, and $\omega$ (columns 1-3, respectively) determine whether or not the community may crash. The solid lines are stable equilibria, the dashed lines unstable equilibria, and the dotted lines mark the bifurcation points. (a) Below the threshold $\iota^{*}$, the community can crash, and above it, it cannot. Here the parameters are $\ell=\omega=\varphi=1$. (b) Above the threshold $\ell^{*}$, the community can crash, and below it, it cannot. Here the parameters are $\iota=0.1$ and $\omega=\varphi=1$. (c) There is a window, between $\omega_{1}^{*}$ and $\omega_{2}^{*}$, in which the community can crash. Outside of this window, it cannot. Here the parameters are $\iota=0.2$ and $\ell=\varphi=1$. F is the CDF of $\mathcal{N}(0.7,0.04)$.

equilibrium disappears and only the polymorphic equilibrium with positive community size is stable. This pattern highlights the potential for hysteresis in the development of a community. For instance, consider a new community with an initially high inflow rate, e.g., because of its novelty or active recruitment efforts by the founders. If this initial inflow is sufficiently high, then the community can become established, and it can survive (albeit at smaller size) even if $\iota$ is reduced over time to level that would have been insufficient to get the community off the ground.

The learning rate $\ell$ has the opposite threshold effect. Below $\ell^{*}$ (Figure 6b), the crashing equilibrium does not exist, and above it, it does. There is again a range of learning rates where both the crashing and polymorphic equilibria are stable. Thus, learning rate also exhibits potential for hysteresis, though in the opposite direction from $\iota$. If initially learning rate can be initially sufficiently suppressed (e.g., by making interactions within the community opaque), the community can emerge and stabilize. Once such a community is established the community will be robust against an increasing $\ell$ (up to a point).

Finally, effect of the leaving rate $\omega$ differs from the inflow and learning rates in that it is non-monotonic (Figure 6c). For low $\omega$, the community size is positive but decreasing in $\omega$ until the crashed equilibrium is the only stable one above a threshold value $\omega_{1}^{*}$. This is intuitive: as individuals leave the community faster, it gets smaller and may not be able to sustain itself. Less intuitive is the fact that there is another threshold, $\omega_{2}$, above 
which the crashed equilibrium is unstable again, and a positive community size is stable. This happens because the high potential leaving rate of savvy individuals initially leave the community mostly composed of naive ones and high cooperation which reduces the realized leaving rate of savvy individuals. This allows the cooperative community to be stable again. These results are similar for the cooperation dilemma (see Appendix SI3). The regions of parameter space where crashing occur are depicted qualitatively in Appendix SI-2.2.

(a)

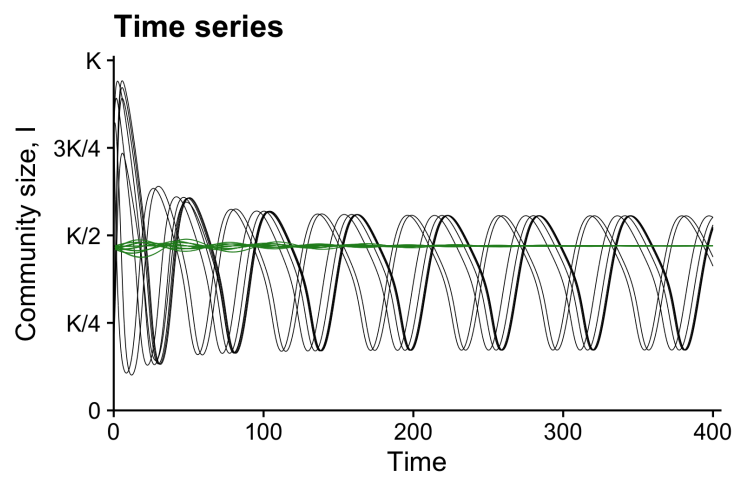

(b)

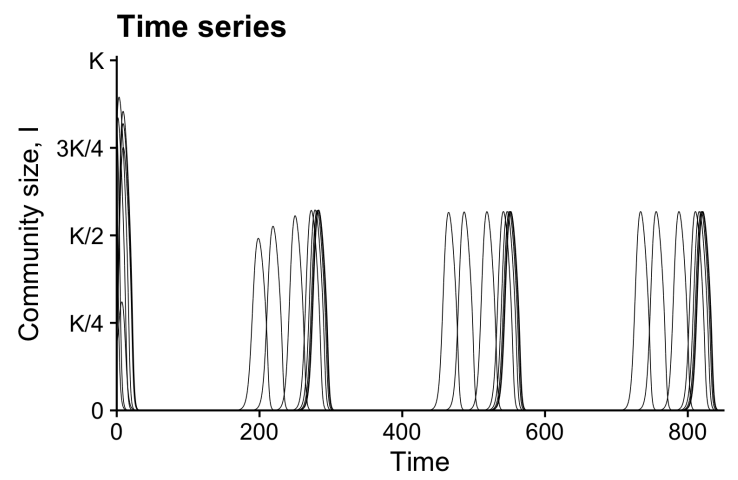

Figure 7: We observe limit cycles for the cooperation dilemma. (a) depicts the time series for $\iota=0.9$ and $\varphi=0.09$. Time series for initial conditions within $1.5 \%$ of the stable equilibrium are plotted in green. Other trajectories that lead to the stable limit cycle are plotted in black. (b) depicts the time series for $\iota=0.5$ and $\varphi=0.009$. For both figures, $\ell=1, \omega=0.85, \hat{q} \approx 0.993$ and $F$ is the CDF of $\mathcal{N}(0.7,0.04)$.

The cooperation dilemma, unlike the coordination case, can also produce an oscillating dynamical regime. Figure 7 depicts an example time series from numerical evaluation of our dynamical equations. In Figure 7a, we observe a bistable regime consisting of a stable equilibrium as well as a limit cycle. On the other hand, Figure $7 \mathrm{~b}$ depicts a case where the community grows and crashes in a relatively short period of time. Between these bursts of activity the community size is minuscule. In this case, all equilibrium points are unstable. See Figure SI-2 in Appendix SI-2.2 for the parameter regions that feature these cycles.

\section{Discussion}

Between the concepts of homo economicus and homo socialis, there is a middle ground where individuals have evolved preferences and acquired social norms that nonetheless are subject to "rational" optimization with constraints (Fehr and Fischbacher, 2002; Gintis. 2007: Akçay et al., 2009; Alger and Weibull, 2013; Gintis, 2014; Morsky and Akçay, 2019). 
Conditional cooperation is an example of this phenomenon. Traxler and Spichtig (Traxler and Spichtig, 2011) showed that conditional cooperation based on norm-dependent relational utilities can sustain cooperation in a community - provided that cooperation is already at a high level. Here, we integrate conditional cooperation norms with a model of community dynamics, and show that cooperation can be built from the ground up by judiciously misrepresenting how much people cooperate. Bootstrapped by rosy prospects of prosociality, prosocial communities can emerge and stabilize, overcoming coordination dilemmas and boosting cooperation. Interestingly, this emergence can be quite abrupt, happening in the form of bifurcations that destroy low-cooperation equilibria. These complicated dynamics can explain sudden changes in population behavior and rise of communities adopting new social norms.

A key aspect of our model is the heterogeneity in how much individuals care about the conditional cooperation norms, as given by the distribution of thresholds for cooperating (Figure 1a). Such heterogeneity has been shown to both hinder and promote cooperation (Heckathorn, 1993: Chatman and Flynn, 2001), and may affect the outcome of interventions to changing harmful social norms such as genital cutting (Efferson et al., 2020). Our results show that the effects of such heterogeneity on community dynamics and cooperation level are complicated. On one hand, heterogeneity can determine whether or not we observe a coordination or cooperation dilemma: for a high mean threshold for cooperating, we have a coordination dilemma with both high and low cooperation equilibria for low heterogeneity vs. a cooperation dilemma with only a low cooperation equilibrium for higher heterogeneity (Figures $4 \mathrm{a}$ and $4 \mathrm{c}$ ). On the other hand, increasing heterogeneity in the coordination dilemma favours cooperation in that it makes the high-cooperation equilibrium globally stable for faster learning (or slower leaving rates; Figure 2b). Yet in cooperation dilemmas, higher heterogeneity moves the high cooperation equilibrium down, and eventually removes the bifurcation that allows high ratio of leaving to learning rates to switch the community to a high-cooperation equilibrium (Figure 4 ).

A paradigm in the cooperation literature is that anonymity hinders cooperation and onymity facilitates it (Wang et al., 2017), as defectors can avoid reciprocity, punishment, or other ill-effects due to their behaviour relative to cooperators, which serve to materially incentivize cooperation. Yet our results illustrate that when most individuals are conditional cooperators (equivalently, conditional defectors), anonymity and ignorance of true behaviour might do better to sustain cooperation. In an onymous version of our model, players could more quickly identify others and their behaviours, thereby learning the true level of cooperation. This points to a potential tradeoff between incentive effects of onymity and the ability of false beliefs to bootstrap cooperation. An interesting ex- 
ample of this trade-off comes from another online community: the file-sharing software Gnutella introduced a feature that allowed users to only share with other sharers. Users could choose to share only with those who were sharing some threshold number of files or more. While this feature may incentivize cooperaiton, it also reveals that the sharing norm is not wide spread and may constrain growth as new users cannot download content. Our model suggests that counter-intuitively, both latter effects will disfavor a cooperative community. Consistent with this, it is unclear if introducing this feature in Gnutella increased cooperation, and may have decreased it (Strahilevitz, 2003).

Our model has interesting implications for strategies to build cooperative communities (or a community following any new norm) in a population of conditional norm followers. We found that the dynamics of community size and cooperation can exhibit hysteresis in important parameters like learning inflow rates: building a cooperative community might require initially high inflow into the community or low learning rates within community, but once a community is established, it can be maintained at lower inflow or higher learning rates. Likewise, we found that the effect of leaving rate is non-monotonic: depending on parameters, the community might exist either with low or high leaving rates but not intermediate. Making it hard to leave the community maintains it straightforwardly, but at the expense of increasing the proportion of savvy individuals, which in the cooperation dilemma reduces overall cooperation levels. On the other hand, making it very easy to leave weeds out the savvy individuals, making it possible for high cooperation levels to build up in the community, after which savvy individuals are less likely to leave. Thus, to build a cooperative community, our model prescribes high recruitment effort, relatively opaque learning opportunities, and easy outs for savvy individuals.

Our model is analogous to compartmental epidemiological models. Our $\mathcal{R}_{0}$, the ratio of entrance to exit rates of the community, plays a fundamental role in determining the presence of polymorphic equilibria of insiders and outsiders as well as whether the community will crash. Our model thus has a place within models of dynamics of communities, including cities, nations, political groups, and other social organizations spanning the small to the large. Further, though we discuss our model within the framework of cooperation, the model applies to any behaviour on which a norm of positive feedback (i.e. the norm prescribes the behaviour the more it is observed). As such, the model has general implications for norm compliance. Examples include the rapid formation of groups, such as opposition to a government, and how they can be facilitated by social media (McGarty et al. 2014). A further example is boom-bust cycles in the housing market. Busts are less likely to happen after a boom if the optimism is justified (as we see in our coordination dilemma) (Burnside et al. 2016). Examples are also found in epidemiology such as confor- 
mity to social distancing and vaccination norms, which have been shown to significantly influence the outcomes of epidemiological models (Reluga, 2010; Oraby et al., 2014).

In this paper, we take the inaccurate initial beliefs of naive individuals as exogenous. Another interesting avenue of research is how such beliefs might form endogenously. It is not difficult to imagine a software company trying to start an active and cooperative community to bias information availability to skew newcomers' beliefs. However, one might also conjecture that such beliefs emerge endogenously if existing community members recruit others promising higher cooperation. Salience bias (Han et al., 2019) can be a consequence of this as cooperation (e.g., making more files available for download) might be more visible than shirking, leading outsiders to overestimate the level of cooperation. Furthermore, recent work by Jackson (2019) shows that in endogenous networks with social interaction, individuals with higher preference for a behaviour form more links, leading to higher visibility of such behaviour. In our model, this would mean that individuals with high relational utility for the cooperation norm would be more active in making links within the community, which would lead to an overestimate of overall cooperation levels. Note that this effect would occur even for savvy individuals, who would have to invest actively to get more representative samples of cooperation to avoid such a bias. That can be a constraint on learning rates in our model. Finally, the false beliefs can also be due to self-interested misrepresentation by community members, who benefit from an influx of newcomers that cooperate more than the existing community members. The potential origins of biased information about cooperation represents an interesting direction for study.

\section{Acknowledgements}

The authors would like to thank the Akçay and Plotkin labs for helpful feedback and discussions.

\section{Author contributions}

Both authors contributed to the conception of the study and wrote the manuscript. B.M. wrote the code and did the mathematical analysis.

\section{Financial support}

This research was supported by the University of Pennsylvania.

\section{Conflicts of interest}

The authors declare that they have no conflicts of interest. 


\section{Data availability}

The code to run the numerical simulations and make figures is available at https:// github.com/bmorsky/communityemergence. For the numerical simulations, we use Julia's DifferentialEquations package (Rackauckas and Nie, 2017).

\section{References}

Erol Akçay and Jeremy Van Cleve. Internalizing cooperative norms in group-structured populations. BioRxiv, page 722439, 2019.

Erol Akçay, Jeremy Van Cleve, Marcus W Feldman, and Joan Roughgarden. A theory for the evolution of other-regard integrating proximate and ultimate perspectives. Proc. Natl. Acad. Sci. USA, 106(45):19061-6, 2009. ISSN 1091-6490. doi: 10.1073/pnas. 0904357106. URL http://www.ncbi.nlm.nih.gov/pubmed/19858492.

Ingela Alger and Jörgen W Weibull. Homo moralis-preference evolution under incomplete information and assortative matching. Econometrica, 81(6):2269-2302, 2013.

Cristina Bicchieri. The Grammar of Society: The Nature and Dynamics of Social Norms. Cambridge: Cambridge University Press, 2006.

Cristina Bicchieri. Norms, preferences, and conditional behavior. Politics, philosophy $\mathcal{E}$ economics, 9(3):297-313, 2010.

Cristina Bicchieri and Alex Chavez. Behaving as expected: Public information and fairness norms. Journal of Behavioral Decision Making, 23(2):161-178, 2010.

Cristina Bicchieri, Eugen Dimant, Simon Gächter, et al. Observability, social proximity, and the erosion of norm compliance. Technical report, CESifo Working Paper No. 8212, 2020a. Available at SSRN: https://ssrn.com/abstract=3576289.

Cristina Bicchieri, Eugen Dimant, and Silvia Sonderegger. It's not a lie if you believe the norm does not apply: conditional norm-following with strategic beliefs. Technical report, Available at SSRN: https://ssrn.com/abstract=3326146 or http://dx.doi.org/10.2139/ssrn.3326146, 2020b.

Craig Burnside, Martin Eichenbaum, and Sergio Rebelo. Understanding booms and busts in housing markets. Journal of Political Economy, 124(4):1088-1147, 2016. 
Jennifer A Chatman and Francis J Flynn. The influence of demographic heterogeneity on the emergence and consequences of cooperative norms in work teams. Academy of management journal, 44(5):956-974, 2001.

Ananish Chaudhuri. Sustaining cooperation in laboratory public goods experiments: a selective survey of the literature. Experimental economics, 14(1):47-83, 2011.

Stephen Coleman. The minnesota income tax compliance experiment: State tax results. Technical report, Minnesota Department of Revenue, 1996.

Charles Efferson, Sonja Vogt, and Ernst Fehr. The promise and the peril of using social influence to reverse harmful traditions. Nature human behaviour, 4(1):55-68, 2020.

Ernst Fehr and Urs Fischbacher. Why social preferences matter - the impact of non-selfish motives on competition, cooperation and incentives. The Economic Journal, 112:1-33, 2002.

Urs Fischbacher, Simon Gächter, and Ernst Fehr. Are people conditionally cooperative? Evidence from a public goods experiment. Economics Letters, 71(3):397-404, June 2001. ISSN 01651765. doi: 10.1016/S0165-1765(01)00394-9.

Bruno S Frey and Stephan Meier. Social comparisons and pro-social behavior: Testing "conditional cooperation" in a field experiment. American Economic Review, 94(5):1717$1722,2004$.

Sergey Gavrilets and Peter J Richerson. Collective action and the evolution of social norm internalization. Proceedings of the National Academy of Sciences, page 201703857, 2017.

Michele J Gelfand and Joshua Conrad Jackson. From one mind to many: the emerging science of cultural norms. Current Opinion in Psychology, 8:175-181, 2016.

Herbert Gintis. The hitchhiker's guide to altruism: Gene-culture coevolution and the internalization of norms. Journal of Theoretical Biology, 220(4):407-418, 2003.

Herbert Gintis. A framework for the unification of the behavioral sciences. The Behavioral and brain sciences, 30(1):1-16; discussion 16-61, 2007. ISSN 0140-525X. doi: 10.1017/ S0140525X07000581. URL http://www.ncbi.nlm.nih.gov/pubmed/17475022.

Herbert Gintis. Social norms as choreography. Politics, Philosophy \& Economics, 9(3):251264, 2010. 
Herbert Gintis. The Bounds of Reason: Game Theory and the Unification of the Behavioral Sciences - Revised Edition. Princeton University Press, 2014.

Michael P Haines. A social norms approach to preventing binge drinking at colleges and universities. Technical report, Newton, MA: Higher Education Center for Alcohol and Other Drug Prevention, Department of Education, 1996.

Bing Han, David Hirshleifer, and Johan Walden. Visibility Bias in the Transmission of Consumption Beliefs and Undersaving. Working paper, University of Toronto, June 2019.

Douglas D Heckathorn. Collective action and group heterogeneity: voluntary provision versus selective incentives. American Sociological Review, pages 329-350, 1993.

Matthew $\mathrm{O}$ Jackson. The friendship paradox and systematic biases in perceptions and social norms. Journal of Political Economy, 127(2):777-818, 2019.

Erik O Kimbrough and Alexander Vostroknutov. Norms make preferences social. Journal of the European Economic Association, 14(3):608-638, 2016.

Melissa A Lewis and Clayton Neighbors. Social norms approaches using descriptive drinking norms education: A review of the research on personalized normative feedback. Journal of American College Health, 54(4):213-218, 2006.

Craig McGarty, Emma F Thomas, Girish Lala, Laura GE Smith, and Ana-Maria Bliuc. New technologies, new identities, and the growth of mass opposition in the a rab s pring. Political Psychology, 35(6):725-740, 2014.

Bryce Morsky and Erol Akçay. Evolution of social norms and correlated equilibria. Proceedings of the National Academy of Sciences, 116(18):8834-8839, 2019.

Tamer Oraby, Vivek Thampi, and Chris T Bauch. The influence of social norms on the dynamics of vaccinating behaviour for paediatric infectious diseases. Proceedings of the Royal Society B: Biological Sciences, 281(1780):20133172, 2014.

H Wesley Perkins. College student misperceptions of alcohol and other drug norms among peers: Exploring causes, consequences, and implications for prevention programs. Designing alcohol and other drug prevention programs in higher education: Bringing theory into practice, pages 177-206, 1997. 
Christopher Rackauckas and Qing Nie. Differentialequations. jl - a performant and feature-rich ecosystem for solving differential equations in julia. Journal of Open Research Software, 5(1), 2017. DOI:http://doi.org/10.5334/jors.151.

Timothy C Reluga. Game theory of social distancing in response to an epidemic. PLoS Comput Biol, 6(5):e1000793, 2010.

Lior Jacob Strahilevitz. Charismatic code, social norms, and the emergence of cooperation on the file-swapping networks. Virginia Law Review, pages 505-595, 2003.

Christian Traxler and Mathias Spichtig. Social norms and the indirect evolution of conditional cooperation. Journal of Economics, 102(3):237-262, 2011.

Zhen Wang, Marko Jusup, Rui-Wu Wang, Lei Shi, Yoh Iwasa, Yamir Moreno, and Jürgen Kurths. Onymity promotes cooperation in social dilemma experiments. Science advances, 3(3):e1601444, 2017.

Shang Xia and Jiming Liu. A belief-based model for characterizing the spread of awareness and its impacts on individuals' vaccination decisions. Journal of The Royal Society Interface, 11(94):20140013, 2014. 


\section{Supplementary Information}

\section{SI-1 Conditional cooperation in a public goods game}

Here we detail the derivation of the threshold behaviour basing the model off of Traxler and Spichtig (2011) with some minor differences. Individuals within the community have a choice to donate to the public good or not. As such, player $i$ may donate at rate $p_{i} \in\{0,1\}$. Those who donate do so at a cost $c>0$. Players' utilities in the community are composed of a material utility and a relational utility, where the material utility is determined by the proportion of cooperation in the community, $\bar{p}=\sum_{i \in N} s_{i} / N$, and all costs incurred by the player, and the relational utility is a function of the difference between the expected material utility of the focal player and their belief about the average material utility earned by the other players within the community. Guilt is a potential relational utility. In the case of file-sharing, users were aware of their own upload to download ratio for some applications (Strahilevitz, 2003). As such, they would know how much they themselves were cooperating relative to their perception of the mean level of cooperation. In some applications, the panel showing this could not be hidden.

The material utility for player $i$ in the community is

$$
u_{i}^{m}\left(p_{i}, \bar{p}\right)=b \bar{p}-c p_{i}
$$

where $b>1$ is the benefit. The relational utility, $u_{i}^{r}\left(p_{i}, q_{i}\right)$, is a function of a player's belief of what others are doing, $q_{i}$, and the action they take, $p_{i}$. We assume that $u_{i}^{r}$ is increasing with respect to $p_{i}$. Let $\theta_{i}$ be a norm sensitivity parameter for player $i$ (we will assume that $\theta_{i} \geq 0$, though it could be negative for antisocial individuals). Then, the total expected utility of player $i$ is

$$
u_{i}\left(p_{i}, q_{i}\right)=b q_{i}-c p_{i}+\theta_{i} u_{i}^{r}\left(p_{i}, q_{i}\right)
$$

Assuming that $u_{i}^{r}$ is increasing with respect to $p_{i}$, a player will donate if $u_{i}\left(1, q_{i}\right)>$ $u_{i}\left(0, q_{i}\right)$, i.e.

$$
\theta_{i}>\frac{c}{u_{i}^{r}\left(1, q_{i}\right)-u_{i}^{r}\left(0, q_{i}\right)} .
$$

Thus, player $i$ can increase their utility by cooperating if they believe that a sufficient number of other players are cooperating (i.e. player $i$ is a conditional cooperator).

Consider a continuous probability distribution of $\theta_{i}$ with cumulative distribution func- 
tion $\Phi(\theta)$. Then, the proportion of individuals that cooperate given some $q_{i}$ is

$$
F\left(q_{i}\right)=1-\Phi\left(\frac{c}{u_{i}^{r}\left(1, q_{i}\right)-u_{i}^{r}\left(0, q_{i}\right)}\right) .
$$

In the main text, we consider $F$ to be the CDF of a normal distribution and that there are two groups with different beliefs, $q_{i}: \bar{p}$ and $\hat{q}$.

\section{SI-2 Analysis of the model}

\section{SI-2.1 Derivation of the model}

The equations for the compartmental model are:

$$
\begin{aligned}
\dot{S} & =\varphi D-\iota(X+Y) \frac{S}{K}, \\
\dot{D} & =\omega(\hat{q}-\bar{p}) Y-\varphi D, \\
\dot{X} & =\iota(X+Y) \frac{S}{K}-\ell(\hat{q}-\bar{p}) X \\
\dot{Y} & =(\hat{q}-\bar{p})(\ell X-\omega Y) .
\end{aligned}
$$

We rewrite our system to be in terms of the number of insiders, $I=X+Y$, and the proportion of savvy insiders, $y=Y / I$. We can determine the dynamics for $I$ as follows:

$$
\dot{I}=\dot{X}+\dot{Y}=\iota I \frac{S}{K}-\ell(\hat{q}-\bar{p}) X+(\hat{q}-\bar{p})(\ell X-\omega Y)=\iota I \frac{S}{K}-\omega(\hat{q}-\bar{p}) y I
$$

And, the dynamics for $y$ can be derived as follows:

$$
\begin{aligned}
\dot{y}=\frac{\dot{Y}}{I}-\frac{Y \dot{I}}{I^{2}} & =(\hat{q}-\bar{p})(\ell(1-y)-\omega y)-\iota y \frac{S}{K}+\omega(\hat{q}-\bar{p}) y^{2} \\
& =(\hat{q}-\bar{p})(1-y)(\ell-\omega y)-\iota y \frac{S}{K} .
\end{aligned}
$$

Combining these equations with $\dot{S}$ and $\dot{p}$ and substituting in $D=K-S-I, I=X+Y$, and $y=Y / I$ gives us: 


$$
\begin{aligned}
& \dot{S}=\varphi(K-S-I)-\iota I \frac{S}{K}, \\
& \dot{I}=\iota I \frac{S}{K}-\omega(\hat{q}-\bar{p}) y I, \\
& \dot{y}=(\hat{q}-\bar{p})(1-y)(\ell-\omega y)-\iota y \frac{S}{K}, \\
& \dot{p}=F(\bar{p})-p .
\end{aligned}
$$

\section{SI-2.2 Stability of equilibria}

Here we discuss the stability conditions for equilibria. To begin with, consider the boundary equilibria (i.e. cases where $I^{*}=0$ or 1 ).

Theorem 1. For the coordination game, the high cooperation equilibrium, $\left(S^{*}, I^{*}, y^{*}, p^{*}\right)=\left(0, K, y^{*} \in\right.$ $[0,1], \hat{q})$ is stable.

Proof. Consider the Jacobian evaluated at this equilibrium,

$$
J^{*}=\left[\begin{array}{cccc}
-\varphi & -\varphi-\iota & 0 & 0 \\
\iota & 0 & 0 & \omega K y^{* 2} \\
-\iota \frac{y^{*}}{K} & 0 & 0 & \left(y^{* 2}-y^{*}\right)\left(\ell-\omega y^{*}\right) \\
0 & 0 & 0 & f(q) y^{*}-1
\end{array}\right]
$$

The eigenvalues are $-\varphi,-\iota, 0$, and $f(q) y^{*}-1 \leq f(q)-1<0$. But for the zero eigenvalue, these are all negative. Further, the centre eigenspace corresponds to the $y$-axis. Thus the equilibrium set is stable.

Theorem 2. If $\mathcal{R}_{0}<1$ and $f\left(\bar{p}^{*}\right)<1$ for $y^{*}$ and $p^{*}$ such that $\left(\hat{q}-\bar{p}^{*}\right)\left(1-y^{*}\right)\left(\ell-\omega y^{*}\right)-\iota y^{*}=0$ and $p^{*}=F\left(\bar{p}^{*}\right)$, then $\left(S^{*}, I^{*}, y^{*}, p^{*}\right)=\left(K, 0, y^{*}, p^{*}\right)$ is attracting.

Proof. Though the model is not defined at $\left(S^{*}, I^{*}\right)=(K, 0)$, we may find $y^{*}$ as the solution to

$$
\begin{aligned}
\lim _{S \rightarrow K} \dot{y} & =(\hat{q}-\bar{p})(1-y)(\ell-\omega y)-\iota y \\
& =\omega(q-p) y^{3}+(\omega(\hat{q}+p-2 q)-\ell(q-p)) y^{2} \\
& +(\ell(2 q-p-\hat{q})-\omega(\hat{q}-q)-\iota) y+\ell(\hat{q}-q)=0
\end{aligned}
$$


Note that by Descartes' rule of signs, if $\omega(\hat{q}+p-2 q)>\ell(q-p)$ and $\ell(2 q-p-\hat{q})>$ $\omega(\hat{q}-q)+\iota$, then there is no positive root. With $y^{*}$ we may find $p^{*}$. The Jacobian evaluated at $\left(K, 0, y^{*}, p^{*}\right)$ is

$$
J^{*}=\left[\begin{array}{cccc}
-\varphi & -\varphi-\iota & 0 & 0 \\
0 & \iota-\omega\left(\hat{q}-\bar{p}^{*}\right) y & 0 & 0 \\
-\frac{\iota y^{*}}{K} & 0 & (q-\hat{q})\left(\omega-\ell y^{*}\right)+\left(p^{*}-q\right)\left(\omega+(\ell-\omega) y^{*}\right) y^{*} & y^{*}\left(y^{*}-1\right)\left(\ell-\omega y^{*}\right) \\
0 & 0 & \left(p^{*}-q\right) f\left(\bar{p}^{*}\right) & y^{*} f\left(\bar{p}^{*}\right)-1
\end{array}\right]
$$

And, the characteristic polynomial of $J^{*}$ is

$$
\operatorname{det}\left(J^{*}-\lambda I\right)=\omega\left(\hat{q}-\bar{p}^{*}\right)(\lambda+\varphi)\left(\lambda+1-\mathcal{R}_{0}\right)\left(\lambda^{2}-\left(j_{33}+j_{44}\right) \lambda+j_{33} j_{44}-j_{34} j_{43}\right),
$$

where $j_{i j} \in J .-\left(j_{33}+j_{44}\right)>0$, since $j_{33}<0$ and $j_{44}<0$. Further, if $f\left(\bar{p}^{*}\right)<1$, then

$$
\begin{aligned}
j_{33} j_{44}-j_{34} j_{43} & =\left((\hat{q}-q)\left(\omega-\ell y^{*}\right)+\left(q-p^{*}\right)\left(\omega+(\ell-\omega) y^{*}\right) y^{*}\right)\left(1-y^{*} f\left(\bar{p}^{*}\right)\right) \\
& -y^{*}\left(1-y^{*}\right)\left(\ell-\omega y^{*}\right)\left(q-p^{*}\right) f\left(\bar{p}^{*}\right) \\
& \geq\left(q-p^{*}\right)\left(\left(\omega+(\ell-\omega) y^{*}\right) y^{*}-\ell\left(1-y+y^{2}\right) y^{*} f\left(\bar{p}^{*}\right)\right) \\
& >\left(q-p^{*}\right)\left(\left(\omega+(\ell-\omega) y^{*}\right) y^{*}-\ell\left(1-y+y^{2}\right) y^{*}\right) \\
& =\left(q-p^{*}\right) y^{*}\left(1-y^{*}\right)\left(\omega+\ell y^{*}\right)>0
\end{aligned}
$$

Therefore, combined with $\mathcal{R}_{0}^{*}<1$, we have that all eigenvalues are negative and thus this state is attracting.

Now consider the internal equilibria (i.e. $I, S \in(0,1)$ ):

$$
\begin{aligned}
S^{*} & =\frac{K}{\mathcal{R}_{0}}, \\
I^{*} & =K \frac{\mathcal{R}_{0}-1}{\mathcal{R}_{0}+\iota / \varphi}, \\
y^{*} & =\frac{\ell}{\ell+\omega}, \\
p^{*} & =F\left(\bar{p}^{*}\right),
\end{aligned}
$$


which exist if $\mathcal{R}_{0}>0$. The Jacobian evaluated at these equilibria is

$$
\begin{aligned}
J^{*} & =\left[\begin{array}{cccc}
-\varphi-\iota \frac{I^{*}}{K} & -\varphi-\iota \frac{S^{*}}{K} & 0 & 0 \\
\iota \frac{I^{*}}{K} & 0 & -\omega\left(\hat{q}-q+2\left(q-p^{*}\right) y^{*}\right) I^{*} & \omega I^{*} y^{* 2} \\
-\iota \frac{y^{*}}{K} & 0 & -(\hat{q}-q)\left(\ell+\omega\left(1-y^{*}\right)\right)-\left(q-p^{*}\right)\left(\ell-2(\ell-\omega) y^{*}\right) y^{*} & -y(1-y)(\ell-\omega y) \\
0 & 0 & -\left(q-p^{*}\right) f\left(\bar{p}^{*}\right) & y^{*} f\left(\bar{p}^{*}\right)-1
\end{array}\right] \\
& =\left[\begin{array}{cccc}
-j_{1} & -j_{2} & 0 & 0 \\
j_{3} & 0 & -j_{4} & j_{5} \\
-j_{6} & 0 & -j_{7} & -j_{8} \\
0 & 0 & -j_{9} & -j_{10}
\end{array}\right] .
\end{aligned}
$$

Here we represent the elements of $J^{*}$ with $j_{i}>0$. Note that for $j_{7}$ we have substituted $\iota S^{*} / K=\omega\left(\hat{q}-\bar{p}^{*}\right) y^{*}$ to simplify the expression. Stability can be determined by the RouthHurwitz criteria. These conditions for our system are:

$$
\begin{aligned}
& a_{3}=j_{1}+j_{7}+j_{10}>0, \\
& a_{2}=j_{2} j_{3}+j_{1} j_{7}+j_{1} j_{10}+j_{7} j_{10}-j_{8} j_{9}>0, \\
& a_{1}=j_{2}\left(j_{4} j_{6}+j_{3}\left(j_{7}+j_{10}\right)\right)+j_{1}\left(j_{7} j_{10}-j_{8} j_{9}\right)>0, \\
& a_{0}=j_{2}\left(j_{6}\left(j_{5} j_{9}+j_{4} j_{10}\right)+j_{3}\left(j_{7} j_{10}-j_{8} j_{9}\right)\right)>0, \\
& a_{1}\left(a_{2} a_{3}-a_{0}\right)-a_{0} a_{3}^{2}>0,
\end{aligned}
$$

where $a_{i}$ are the coefficients of the characteristic polynomial $P(\lambda)=\sum_{0}^{n} a_{n} \lambda^{n}$ of $J^{*}$. Note that

$$
\begin{aligned}
a_{0} & =j_{2}\left(\left(j_{5} j_{6}-j_{3} j_{8}\right) j_{9}+\left(j_{4} j_{6}+j_{3} j_{7}\right) j_{10}\right) \\
& =j_{2}\left(\left(\omega y^{* 2}-\left(1-y^{*}\right)\left(\ell-\omega y^{*}\right)\right) \frac{\iota I^{*} y^{*} j_{9}}{K}+\left(j_{4} j_{6}+j_{3} j_{7}\right) j_{10}\right) \\
& =j_{2}\left(\left(\frac{\ell \omega}{\ell+\omega}-\frac{\ell \omega}{\ell+\omega}\right) \frac{\iota I^{*} y^{*} j_{9}}{K}+\left(j_{4} j_{6}+j_{3} j_{7}\right) j_{10}\right) \\
& =j_{2}\left(j_{4} j_{6}+j_{3} j_{7}\right)\left(1-y^{*} f\left(\bar{p}^{*}\right)\right) .
\end{aligned}
$$

If $y^{*} f\left(\bar{p}^{*}\right) \geq 1$, then $a_{0} \leq 0$ and the state cannot be stable. And, so long as $y^{*} f\left(\bar{p}^{*}\right)<1$, the equilibrium can be stable. Note that this is a necessary, but not sufficient, condition.

We numerically explored the existence and stability of equilibria across parameter 
(a)

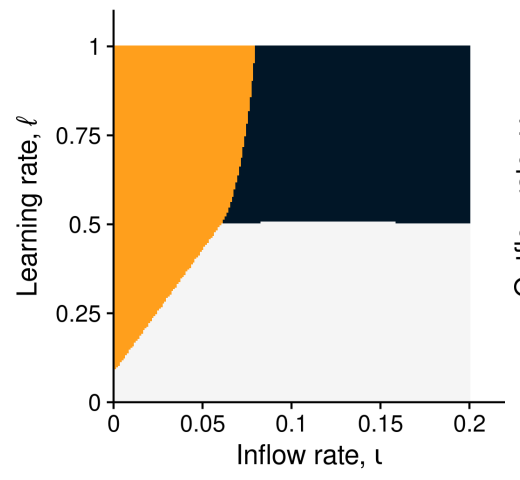

(d)

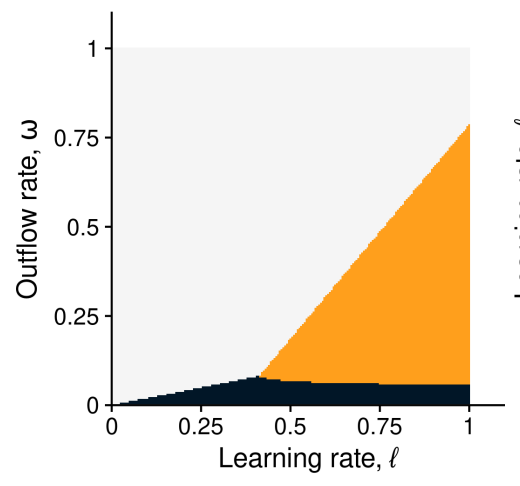

(b)

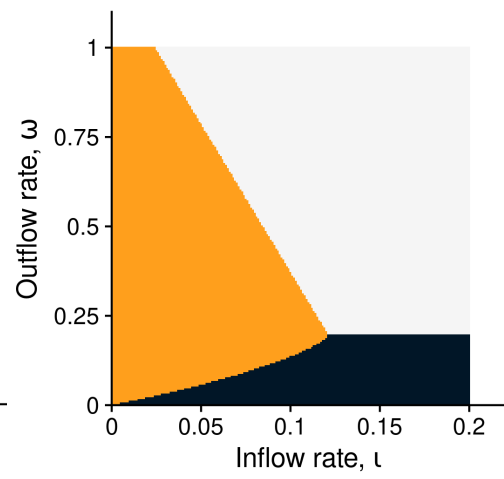

(c)

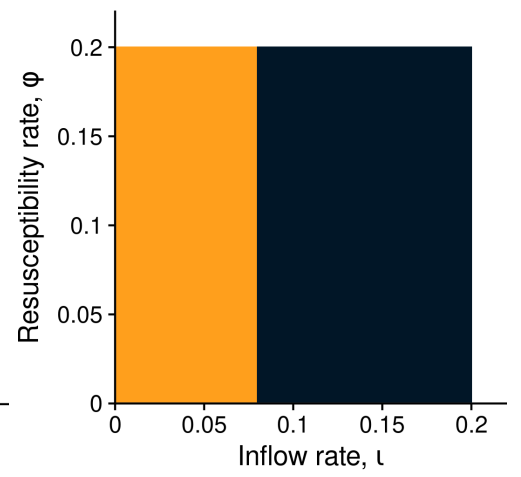

(f)

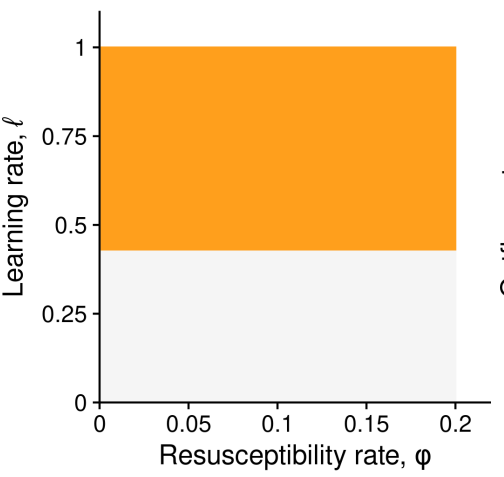

Nature of equilibria: $\quad 1$ stable $\square 2$ stable stable $\&$ crash

Figure SI-1: Here we plot the qualitative behaviour of the coordination dilemma model across parameter space. We observe the stable high cooperation equilibrium either alone (in grey), with a stable interior point (black), and with a stable crash equilibrium (orange). The default values are $\iota=\varphi=0.05, \ell=1$, and $\omega=0.1$. These give us $\omega / \ell=0.1$, where the two interior equilibria can exist. Stability is determined by the Routh-Hurwitz criteria (Inequalities SI.25. SI.29) and Theorem SI-3.

space using the Routh-Hurwitz criteria (Inequalities SI.28 SI.29) and Theorem 2, Figures SI-1 and SI-2 summarize the impact of the parameters, qualitatively, for the coordination and cooperation dilemmas, respectively. For the coordination dilemma, we observe that increasing $\ell$ can result in the emergence of interior equilibria as well as the system crashing from the single high cooperation equilibrium (Figure SI-1a and SI-1d). $\omega$ has the opposite effect; decreasing it can result in the emergence of such equilibria (Figure SI-1b and SI-1d). Notice that for some areas of parameter space, we must traverse the regime with crashing to move from the two non-crashing stable equilibria regime to the sole high cooperation stable equilibrium regime. Varying $\varphi$ has no effect on the qualitative nature of the system here. 
(a)

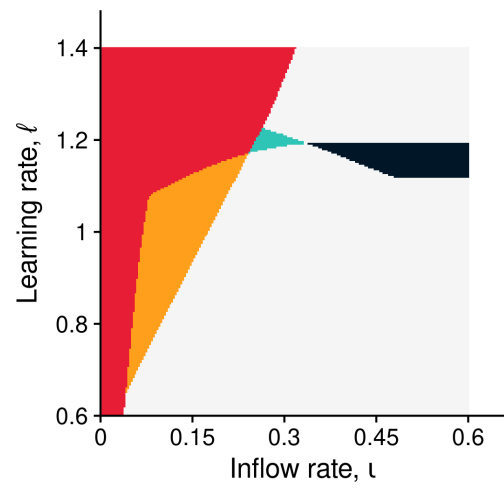

(d)

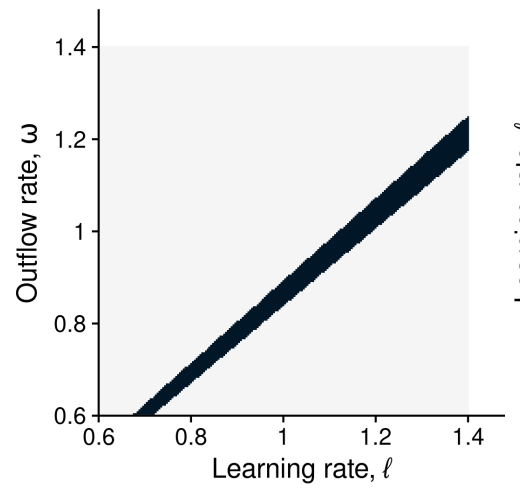

(b)

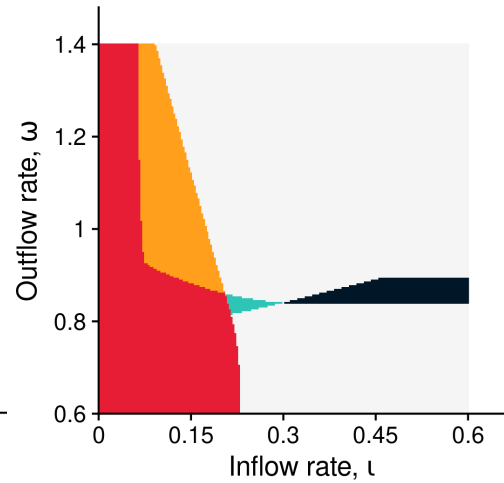

(c)

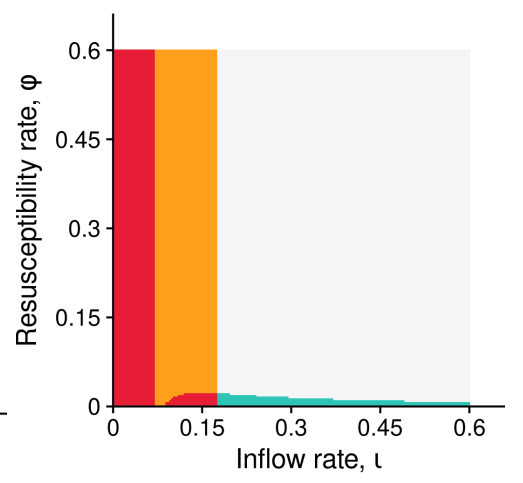

(e)

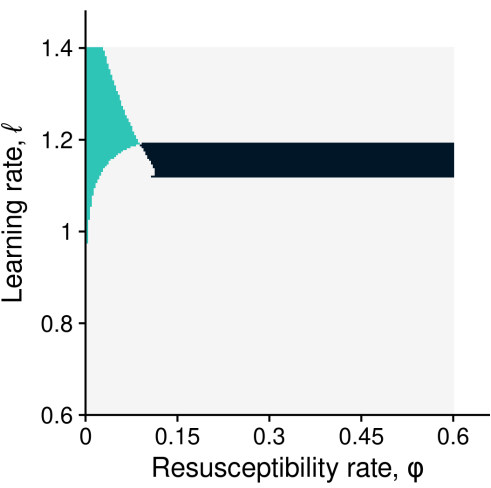

(f)

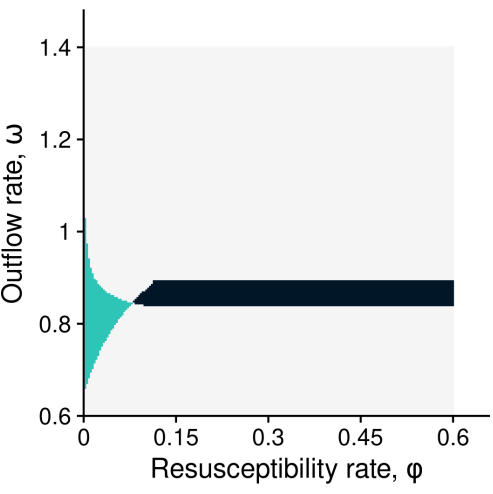

Nature of equilibria: cycle 1 stable $\square 2$ stable crash stable \& crash

Figure SI-2: Here we plot the qualitative behaviour of the cooperation dilemma model across parameter space. We observe cycling (turquoise), one stable interior equilibrium either (grey), two stable interior equilibria (black), only the crashing equilbirium (red), and stable interior and crashing equilibria (orange). The default values are $\iota=\varphi=0.5$ and $\ell=\omega=1$. Stability is determined by the Routh-Hurwitz criteria (Inequalities SI.25, SI.29) and Theorem SI-3

For the cooperation dilemma, Figure SI-2, we observe a variety of affects of the parameters on the qualitative behaviours the system can have. Increasing $\iota$ and decreasing $\ell$ can pull us from regimes that feature crashing to ones that do not (Figures SI-2a.SI-2c). The intuition behind these results comes from noting that $\mathcal{R}_{0}$ increases/decreases with respect to $\iota / \ell$. Varying $\iota$ and $\ell$ can also take us through two other regimes, namely the cycling and two stable interior equilibria regimes. In the cycling regime, we find that no equilibria are stable. We verified through numerical simulations that we observe cycling here. Our figures do not, however, show all regions where cycling can occur. For example, we can have a cycle along with a stable fixed point as shown in Figure $7 \mathrm{a}$ of the main manuscript, and thus cycling is possible in the other regimes depicted here.

We discussed the roles of $\ell$ and $\omega$ on the community size in Figure 5 of the main 
manuscript. In Figure SI-2d, we see that the two stable equilibria regime is observed for intermediate values of $\ell$ vs. $\omega$. For higher or lower values of these parameters, we only observe one stable equilibrium. Finally, in Figures SI-2e and SI-2f. we observe that reducing $\varphi$ can result in cycling when $\omega / \ell$ is low. However, if $\omega / \ell$ is too low, we will have a stable interior equilibrium.

\section{SI-3 Community crashing in the coordination dilemma}

(a)

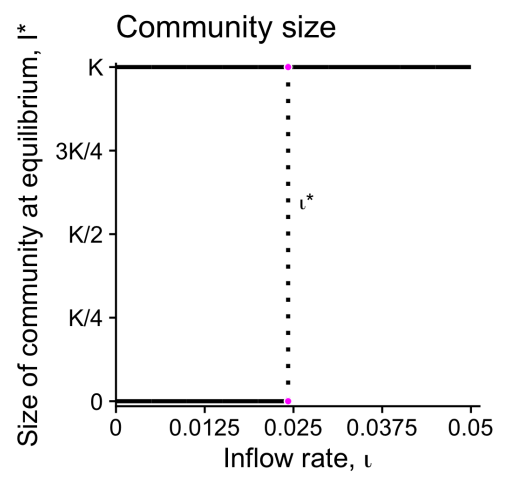

(b)

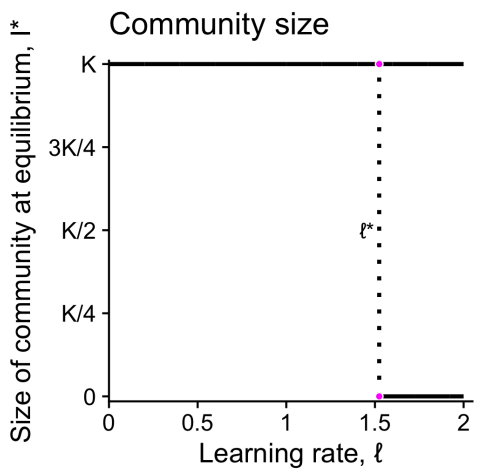

(c)

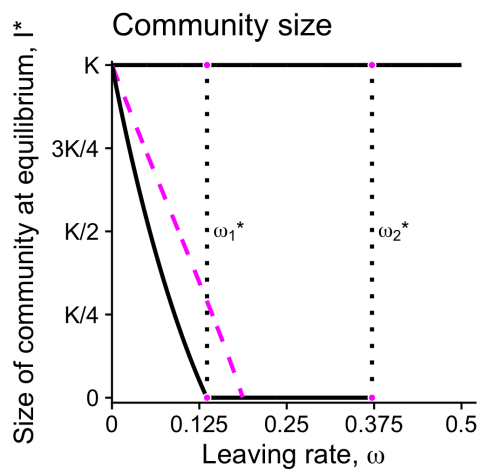

Figure SI-3: The parameters $\iota, \ell$, and $\omega$ (columns 1-3, respectively) determine whether or not the community may crash. The solid lines are stable equilibria, the dashed lines unstable equilibria, and the dotted lines mark the bifurcation points. (a) Below the threshold $\iota^{*}$, the community can crash, and above it, it cannot. Here the parameters are $\ell=\omega=\varphi=1$. (b) Above the threshold $\ell^{*}$, the community can crash, and below it, it cannot. Here the parameters are $\iota=0.1$ and $\omega=\varphi=1$. (c) There is a window, between $\omega_{1}^{*}$ and $\omega_{2}^{*}$, in which the community can crash. Outside of this window, it cannot. Here the parameters are $\iota=0.1$ and $\ell=\varphi=1$. F is the CDF of $\mathcal{N}(0.5,0.04)$.

Figure SI-3 plots the effects of different parameters on the existence of the crashing state for the coordination dilemma. We observe similar qualitative results to the cooperation dilemma; the community crashes for sufficiently low inflow, high learning, and intermediate outflow rates. Our observation on hysteresis is also applicable here, and more dramatic though less surprising as this is a coordination dilemma. One noticeable difference between the two dilemmas, however, is that community crashing cannot be globally stable here (due to Theorem 1 ). 